\title{
Paths to equilibrium in non-conformal collisions
}

\author{
Maximilian Attems ${ }^{1,2, \star}$, Yago Bea ${ }^{1}$, Jorge Casalderrey-Solana ${ }^{3}$, David Mateos ${ }^{1,4}$, Daniel Santos- \\ Oliván $^{5}$, Carlos F. Sopuerta ${ }^{5}$, Miquel Triana ${ }^{1}$, and Miguel Zilhão ${ }^{1,6}$, \\ ${ }^{1}$ Departament de Física Quàntica i Astrofísica \& Institut de Ciències del Cosmos (ICC), Universitat de \\ Barcelona, Martí i Franquès 1, 08028 Barcelona, Spain \\ ${ }^{2}$ Instituto Galego de Física de Altas Enerxías (IGFAE), Universidade de Santiago de Compostela, 15782 \\ Galicia, Spain \\ ${ }^{3}$ Rudolf Peierls Centre for Theoretical Physics, University of Oxford, 1 Keble Road, Oxford OX1 3NP, United \\ Kingdom \\ ${ }^{4}$ Institució Catalana de Recerca i Estudis Avançats (ICREA), Passeig Lluís Companys 23, 08010, Barcelona, \\ Spain \\ ${ }^{5}$ Institut de Ciències de l'Espai (CSIC-IEEC), Campus UAB, Carrer de Can Magrans s/n, 08193 Cerdanyola \\ del Vallès, Spain \\ ${ }^{6}$ CENTRA, Departamento de Física, Instituto Superior Técnico, Universidade de Lisboa, Avenida Rovisco \\ Pais 1, 1049 Lisboa, Portugal
}

\begin{abstract}
Ever since fast hydrodynamization has been observed in heavy ion collisions the understanding of the hot early out-of-equilibrium stage of such collisions has been a topic of intense research. We use the gauge/gravity duality to model the creation of a strongly coupled Quark-Gluon plasma in a non-conformal gauge theory. This numerical relativity study is the first non-conformal holographic simulation of a heavy ion collision and reveals the existence of new relaxation channels due to the presence of non-vanishing bulk viscosity. We study shock wave collisions at different energies in gauge theories with different degrees of non-conformality and compare three relaxation times which can occur in different orderings: the hydrodynamization time (when hydrodynamics becomes applicable), the EoSization time (when the average pressure approaches its equilibrium value) and the condensate relaxation time (when the expectation value of a scalar operator approaches its equilibrium value). We find that these processes can occur in several different orderings. In particular, the condensate can remain far from equilibrium even long after the plasma has hydrodynamized and EoSized.
\end{abstract}

\section{Motivation}

The gauge/gravity duality entered a new area with the prediction of the low viscosity over sheer entropy ratio [1] for $\mathcal{N}=4$ Super-Yang-Mills plasma. It turned out that the strongly coupled Quark Gluon plasma (QGP) created in ultra-relativistic heavy-ion collisions features transport properties of such an almost perfect liquid [2,3]. Fast Hydrodynamization, meaning the early applicability of hydrodynamics to such a liquid, got established first in boost invariand flow and than by colliding

${ }^{\star}$ Speaker, e-mail: attems@icc.ub.edu 
holographic shock waves [4-10], which trigger out-of-equilibrium strongly coupled dynamics. These numerical studies were extended to non-conformal models [11, 12], where the fast applicability to hydro was confirmed for non-conformal gauge theories [13-15]. EoSization, the time when the ideal equation of state applies again, was found to depend on bulk viscosity. Moreover see the lattice study [16] using holography for probing the Quantum ChromoDynamics (QCD) phase diagram at finite baryon chemical potential. For a full introduction to holography and a review over it's application to the QGP created in collisions at the Relativistic Heavy Ion Collider and at the Large Hadron Collider we refer the reader to the book review [17].

Shockwave collisions in an asymptotically AdS spacetime, have provided insights into the farfrom-equilibrium properties of hot, strongly-coupled, non-Abelian plasmas that are potentially relevant for the QGP. The dynamics of the longitudinal, transverse and average pressures provides information about different relaxations in the evolving plasma. The non-conformality of the plasma involves an additional channel, namely the evolution of the energy density and the average pressure towards asymptotic values related by the equation of state. This process is named "EoSization" and once it happened the plasma has "EoSized". As EoSization is in part controlled by how the condensate approaches its equilibrium we show new scalar condensate evolution. We expand our previous results of the non-conformal colliding shockwaves in this proceedings for the Lattice 2017 conference in Granada, Spain.

\section{Setup}

The action with $\kappa_{5}$ the five-dimensional Newton constant for our minimally coupled Einstein-plusscalar models [13] is

$$
S=\frac{2}{\kappa_{5}^{2}} \int d^{5} x \sqrt{-g}\left[\frac{1}{4} \mathcal{R}-\frac{1}{2}(\nabla \phi)^{2}-V(\phi)\right] .
$$

The holographic renormalization procedure [18] for the scalar potential $V(\phi)$ fixes the first terms on top of the negative cosmological constant of anti-de-Sitter (AdS) space. This leaves the freedom in the higher terms of the potential for a single-parameter $\phi_{\mathrm{M}}$, which dictates the degree of non-conformality of the given theory. The renormalization group flow between the two fixed points in the ultra-violet (UV) and the infra-red explicitly breaks conformal invariance. The dual operator of the scalar field has in the UV mass dimension three. We emphasize that our choice of potential is not guided by the desire to mimic detailed properties of QCD but by simplicity:

$$
L^{2} V(\phi)=-3-\frac{3}{2} \phi^{2}-\frac{1}{3} \phi^{4}+\left(\frac{1}{2 \phi_{\mathrm{M}}^{4}}+\frac{1}{3 \phi_{\mathrm{M}}^{2}}\right) \phi^{6}-\frac{1}{12 \phi_{\mathrm{M}}^{4}} \phi^{8} .
$$

$L$ is the the radius of the corresponding AdS solution in the UV. In the Fefferman-Graham metric one gets a quasi-analytic solution for a single traveling shockwave on a vacuum background, the seminal Aichelburg-Sexl solution [19]. $u_{\mathrm{FG}}$ is the holographic coordinate, $x_{ \pm}$the propagation direction of the shockwave and $x_{\perp}$ the perpendicular directions to it. The metric Ansatz corresponds to the vacuum metric plus the addition of a Gaussian function term $h\left(x_{ \pm}\right)$, where $f\left(u_{F G}\right)$ is a second-order differential equation coming from the Einstein equation coupled to the potential:

$$
d s^{2}=\frac{L^{2}}{u_{\mathrm{FG}}^{2}} d u_{\mathrm{FG}}^{2}+f\left(u_{\mathrm{FG}}\right) h\left(x_{ \pm}\right) d x_{ \pm}^{2}+e^{2 a_{\mathrm{FG}}\left(u_{\mathrm{FG}}\right)}\left(-d x_{+} d x_{-}+d \mathbf{x}_{\perp}^{2}\right),
$$


From the vacuum solution for the chosen potential one determines $a_{\mathrm{FG}}$ and numerically solves $f\left(u_{\mathrm{FG}}\right)$ (see [14] for the detailed procedure). We choose for the gravitational wave the Gaussian with the width $\omega$ of

$$
h\left(z_{ \pm}\right)=\frac{\mu^{3}}{w \sqrt{2 \pi}} e^{-z_{ \pm}^{2} / 2 \omega^{2}},
$$

where $\mu \omega$ parametrizes the dimensionless "thickness" of the chosen initial profile.

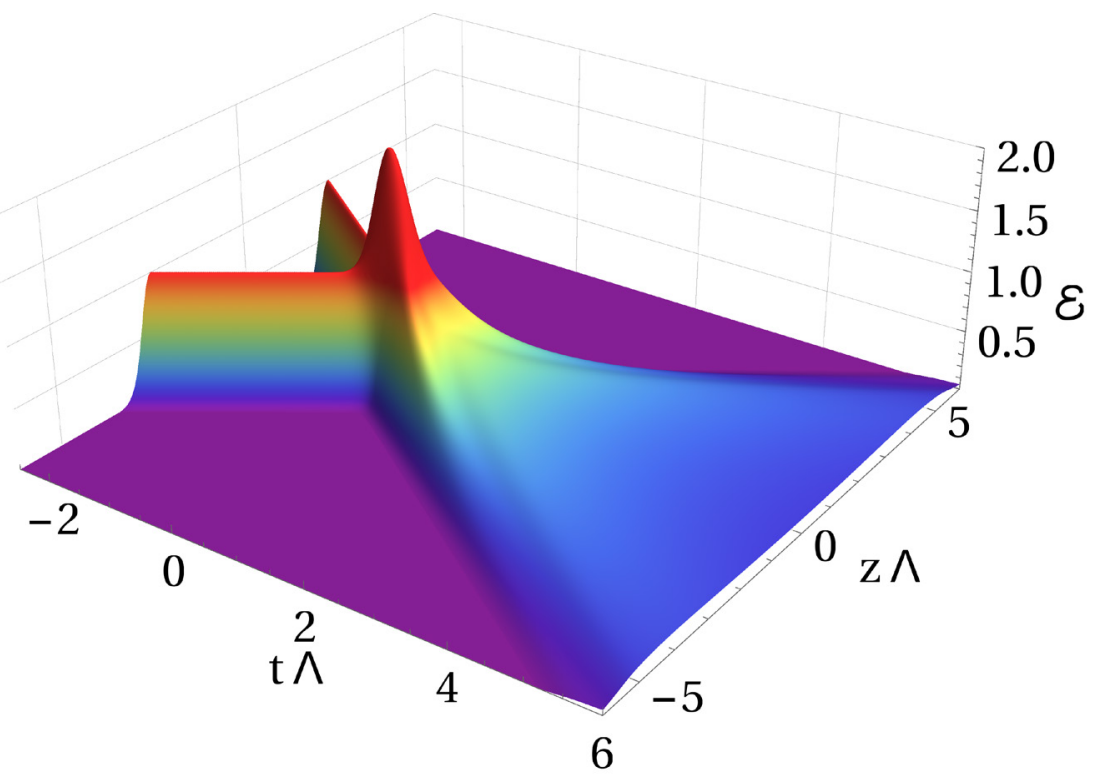

Figure 1. Spacetime evolution of the non-conformal shockwaves, in units of $\Lambda^{4}$, in a collision in the $\phi_{M}=20$ model with $\mu \omega=0.30$ and $\mu / \Lambda=0.93$.

The Ward identity for the trace of the stress tensor gives

$$
\left\langle T_{\mu}^{\mu}\right\rangle=-\Lambda\langle O\rangle
$$

where $\Lambda$ controls the magnitude of the non-normalizable mode of the scalar field and is the source of the conformal invariance breaking. Omitting the expectation value signs we will work with the rescaled quantities of the energy momentum tensor

$$
\left(\mathcal{E}, P_{L}, P_{T}, \mathcal{V}\right)=\frac{\kappa_{5}^{2}}{2 L^{3}}\left(-T_{t}^{t}, T_{z}^{z}, T_{x_{\perp}}^{x_{\perp}}, O\right) .
$$

In these variables the Ward identity is given by

$$
\mathcal{E}-3 \bar{P}=\Lambda \mathcal{V} .
$$

with

$$
\bar{P}=\frac{1}{3}\left(P_{L}+2 P_{T}\right)
$$

The scalar expectation value $\mathcal{V}=0$ is null for the vacuum according to the chosen renormalization. Out of equilibrium it fluctuates independently. 


\section{Non-conformal collisions}

\subsection{Hydrodynamization and EoSization}

The physics of the resulting collision depends on the "thickness" eq. (4) of the collision and on the initial transverse energy density in units of the source $\mu / \Lambda$. In Figure 1 we show the spacetime evolution of a very non-conformal collision $[13,14]$. From a birds eye view the evolution seems quite similar to the ones of conformal collisions, but its pressure evolution differs. In the terminology of [7] this is a $1 / 2$-shock with $\mu \omega=0.30$. We extract the hydrodynamization time $t_{\text {hyd }}$ according to the time when the following criteria of first-order hydrodynamics matches to better than $10 \%$ :

$$
\left|\frac{P_{L, T}-P_{L, T}^{\mathrm{h} y d}}{\bar{P}}\right|<0.1
$$

$\bar{P}$ is the average pressure. EoSization $t_{\mathrm{E} o S}$ is the time beyond which the normalized average pressure agrees with the equilibrium pressure with a $10 \%$ accuracy:

$$
\left|\frac{\bar{P}-P_{\mathrm{e} q}}{\bar{P}}\right|<0.1
$$

Note that we used the average pressure to normalize (in a CFT the local energy density has a trivial relation to pressure and hence is often in the denominator).

In our temperature scan in Figure 2 we extract for each collision the hydrodynamization time (red dashed line) and the EoSization time (purple dashed line). Analysing it one finds an increase with non-conformality of the hydrodynamization time. The increase of $t_{\text {hyd }} T_{\text {hyd }}$ is at maximum a factor of 2.6 compared to the CFT result. Moreover the EoSization time happens after the hydrodynamization where as in a CFT the plasma is EoSized at all times per definition.

For sufficiently large $\mu / \Lambda$ we see indeed EoSization becoming negative in Figure 2, meaning that the average and the equilibrium pressures differ by less than $10 \%$ even before the shocks collide. This is due to the start of an overlap of the shocks before the collision and shows that those collisions are almost conformal.

Both non-conformal effects are understood as a consequence of the presence of bulk viscosity, which is shown in function of temperature in the upper panel of Figure 2. The bulk viscosity reduces the speed of the expansion of the plasma. The maximal hydrodynamization time $t_{\text {hyd }} T_{\text {hyd }}$ is reached with a bulk viscosity over sheer viscosity ratio of $\zeta / s \equiv 0.029$. Indeed one only needs a bulk viscosity over sheer viscosity of $\zeta / s>0.025$ to get sizable non-conformality for the early non-equilibrium evolution. We extract this value as a conservative estimate below which EoSization always happens before hydrodynamization. This is exciting as this may well be below the range of the estimated QCD bulk viscosity from the temperature of the critical point up to twice its value, which has not yet been computed convincingly with lattice QCD. This is the temperature range of the RHIC collisions in the search of the critical point. The bulk viscosity is thought to have a strong effect $[20,21]$ on the observable of the QCD phase transition. 


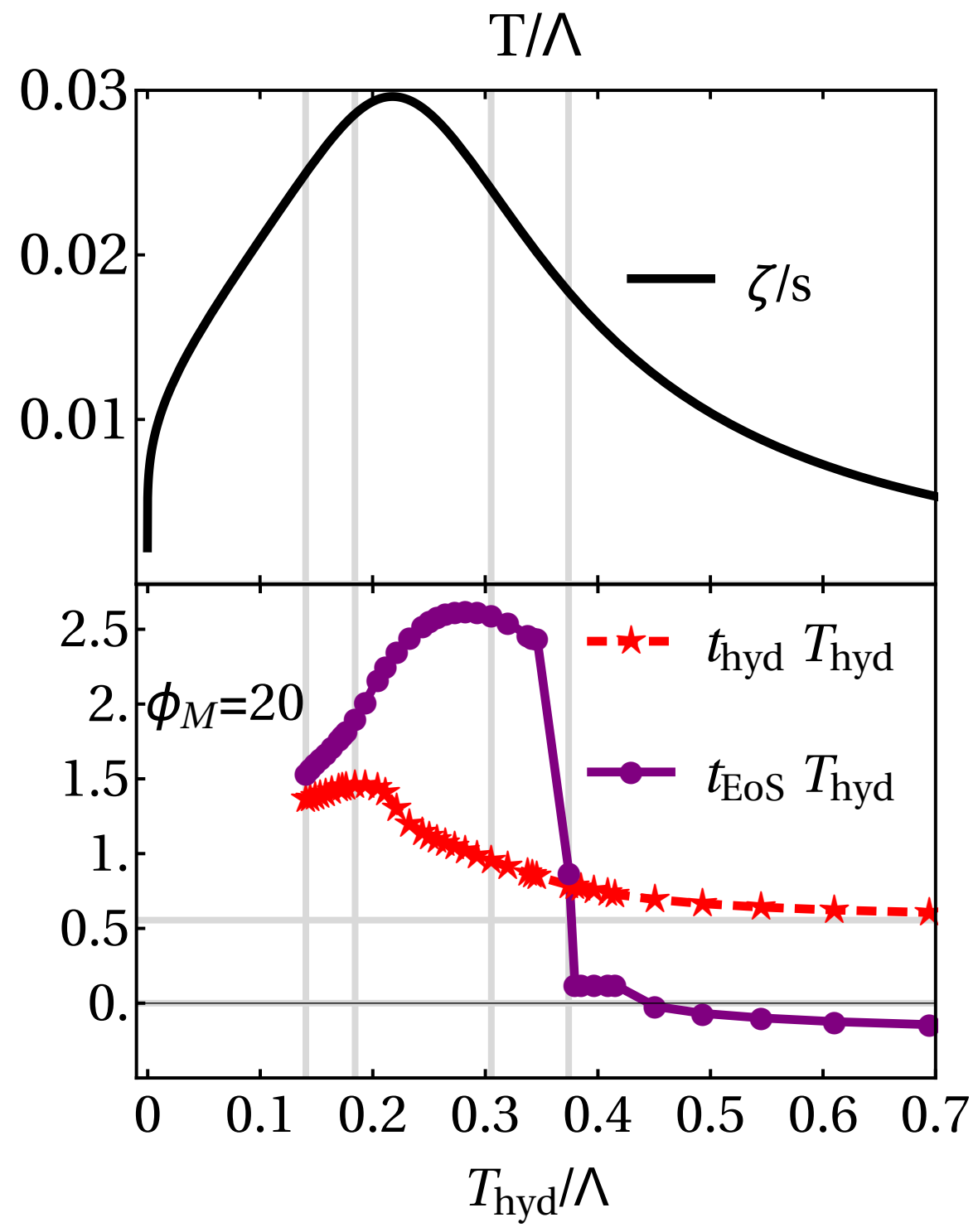

Figure 2. (upper panel) Ratio of bulk viscosity $\zeta$ over entropy $\eta$ in function of temperature for $\phi_{M}=20$. (lower panel) Hydrodynamization (red dotted line) and EoSization times (purple full line) as a function of the hydrodynamization temperature for collisions of shocks with $\mu \omega=0.32$ for $\phi_{M}=20$. The horizontal grey line lies at $t_{\mathrm{h} y d} T_{\mathrm{h} y d}=0.56$ and corresponds to the CFT limit. From left to right, the four vertical grey lines indicate the hydrodynamization temperatures for the collisions with the minimal value of $T_{\text {hyd }} / \Lambda$, the maximum value of $t_{\text {hyd }} T_{\text {hyd }}$, the maximum value of the ratio $t_{\text {Eos }} / t_{\text {hyd }}$ and the high-temperature crossing at which $t_{\text {EoS }}=t_{\text {hyd }}$. 


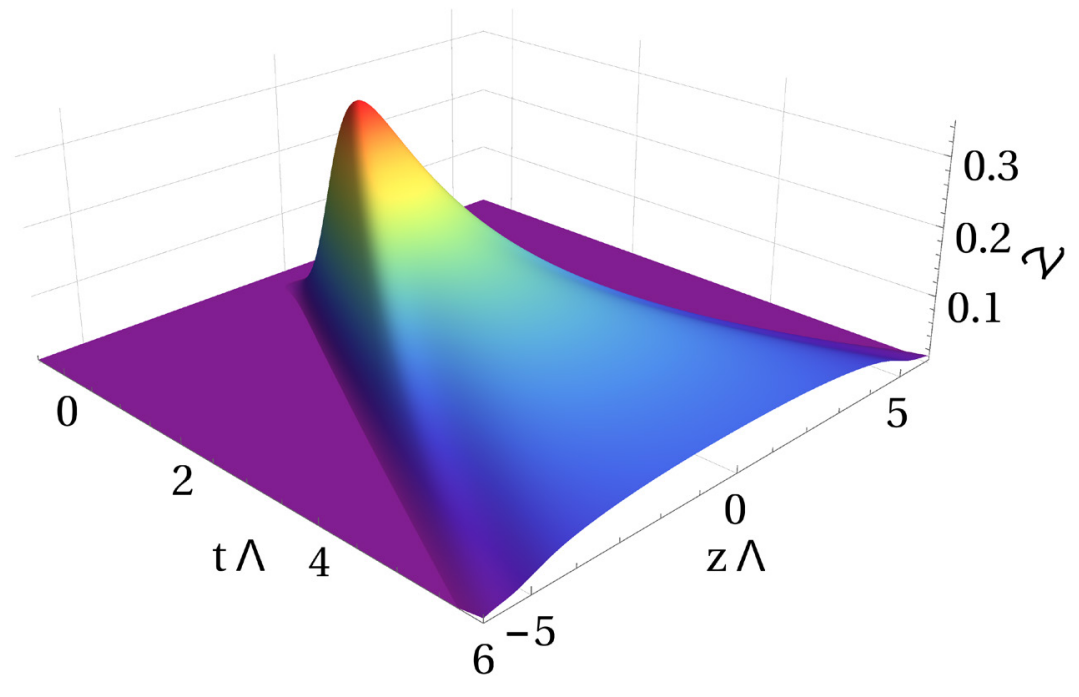

Figure 3. Spacetime evolution of the scalar condensate, in units of $\Lambda^{3}$, in a collision in the $\phi_{M}=20$ model with $\mu \omega=0.30$ and $\mu / \Lambda=0.93$.

\subsection{Scalar condensate relaxation}

In analogy to the hydrodynamization and EoSization times we define a criterium for the condensate relaxation time. It is fullfilled once the normalized difference of the expectation value and its equilibrium value

$$
\left|\frac{\mathcal{V}-\mathcal{V}_{\mathrm{e} q}}{\mathcal{V}}\right|<0.1
$$

differ by less than $10 \%$ at any later time. It indicates how fast this one-point function reaches its equilibrium value. The condensate relaxation time for the specific collision of Figure 1 is shown in Figure 3 happens to be last after both hydrodynamization and EoSization times. For more nonconformal setups the condensate relaxation can happen first. Figure 4 shows the scalar condensate evolution of such an outcome. As expected in all the evolution the scalar condensate is not excited before the collision and sharply peaks afterwards. For collisions in the non-conformal temperature range the scalar condensate is fully out of equilibrium. The decay of the scalar condensate happens in an oscillating way for the lower temperature collisions as governed by the intermixed quasinormal modes of the theory.

One notes of course the delay of the maximal peak in the scalar condensate (see Figures 3,4) compared to the local energy density superposition of the shocks (see Figure 1). This delay is also observed in e.g. the true drag force on a quark compared to the force that it would experience in an equilibrium plasma with the same instantaneous energy density [22,23].

It is remarkable how hydrodynamics works so well describing a non-conformal plasma even with a non-trivial equation of state. This success is even more surprising in cases in which hydrodynamization precedes all other equilibration processes. What is perhaps most surprising is that, as measured 


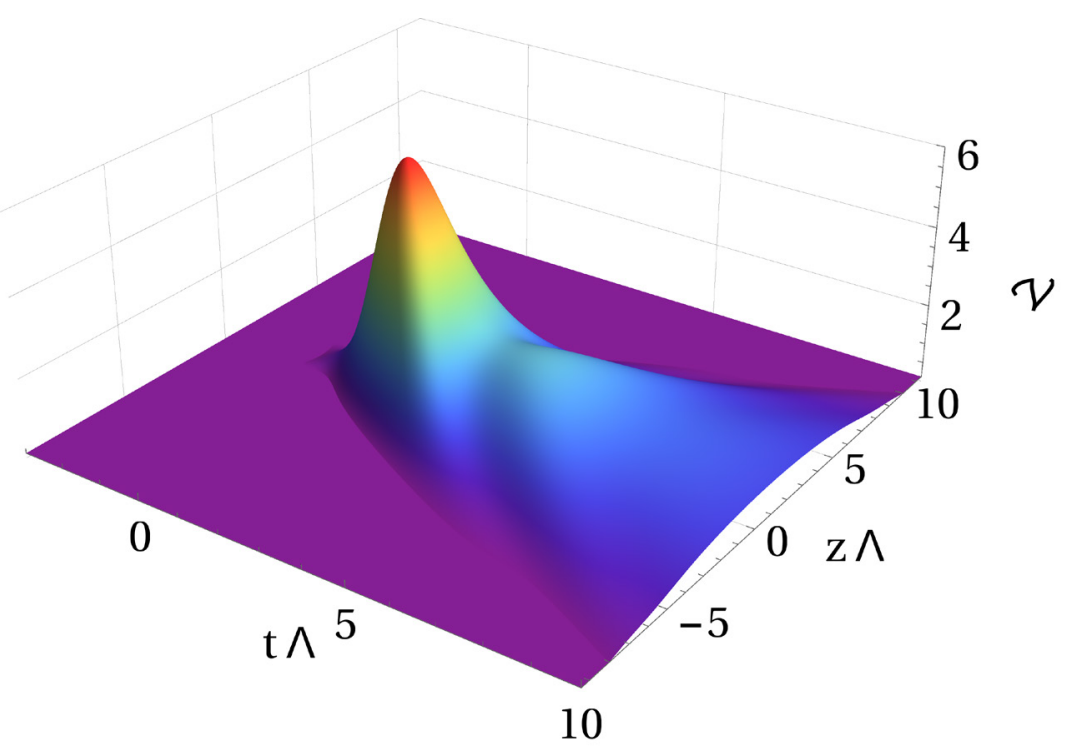

Figure 4. Spacetime evolution of the scalar condensate, in units of $\Lambda^{3}$, in a collision in the $\phi_{M}=20$ model with $\mu \omega=0.30$ and $\mu / \Lambda=0.36$.

by the bulk viscosity-to-entropy ratio, a "sufficient" degree of non-conformality requires only a small value $\zeta / s \gtrsim 0.025$, as first estimated in [13]. This indicates that non-conformal relaxation may also occur in real-world heavy ion collisions, where both calculations [24-26] and data-driven parametrization [27-29] yield larger values than this estimate in a significant part of the time evolution of the resulting plasma. It will be interesting to extend this non-conformal holographic study to models with finite chemical potential [30, 31] or with a phase transition [15].

\section{Acknowledgments}

We thank R.G. Jha, M. Laine, F. Mescia, G. Moore, A. Ohnishi, E. Shuryak for discussions. We thank the supercomputer MareNostrum at the Barcelona Supercomputing Center for providing computational resources (project no. ub65). The work of MA has been supported by a Marie SklodowskaCurie Individual Fellowship of the European Commission's Horizon 2020 Programme under contract number 658574 FastTh. JCS is a Royal Society University Research Fellow. JCS was also supported by a Ramón y Cajal fellowship, by the Marie Curie Career Integration Grant FP7-PEOPLE2012-GIG-333786 and by the Spanish MINECO through grant FPA2013-40360-ERC. CFS and DS acknowledge the support from contracts ESP2013-47637-P and ESP2015-67234-P (Spanish Ministry of Economy and Competitivity of Spain, MINECO). MZ acknowledges support through the FCT (Portugal) IF programme, IF/00729/2015. We also acknowledge funding from grants MEC FPA201346570-C2-1-P, MEC FPA2013-46570-C2-2-P, MDM-2014-0369 of ICCUB, 2014-SGR-104, 2014SGR-1474, CPAN CSD2007-00042 Consolider-Ingenio 2010, ERC grant HotLHC ERC-2011-StG279579, by Ministerio de Ciencia e Innovacion of Spain under project FPA2014-58293-C2-1-P; and by Xunta de Galicia (Conselleria de Educacion) and FEDER; IGFAE is a Maria de Maetzu Excellence Unit (grant number MDM-2016-0692) and ERC Starting Grant HoloLHC-306605. 


\section{References}

[1] G. Policastro, D.T. Son, A.O. Starinets, Phys. Rev. Lett. 87, 081601 (2001), hep-th/0104066

[2] P. Romatschke, U. Romatschke, Phys.Rev.Lett. 99, 172301 (2007), 0706. 1522

[3] R.D. Weller, P. Romatschke, Phys. Lett. B774, 351 (2017), 1701.07145

[4] P.M. Chesler, L.G. Yaffe, Phys. Rev. D82, 026006 (2010), 0906.4426

[5] M.P. Heller, R.A. Janik, P. Witaszczyk, Phys. Rev. Lett. 108, 201602 (2012), 1103.3452

[6] P.M. Chesler, L.G. Yaffe, Phys.Rev.Lett. 106, 021601 (2011), 1011.3562

[7] J. Casalderrey-Solana, M.P. Heller, D. Mateos, W. van der Schee, Phys.Rev.Lett. 111, 181601 (2013), 1305.4919

[8] J. Casalderrey-Solana, M.P. Heller, D. Mateos, W. van der Schee, Phys. Rev. Lett. 112, 221602 (2014), 1312.2956

[9] P.M. Chesler, L.G. Yaffe, JHEP 10, 070 (2015), 1501 . 04644

[10] P.M. Chesler, Phys. Rev. Lett. 115, 241602 (2015), 1506.02209

[11] A. Buchel, M.P. Heller, R.C. Myers, Phys. Rev. Lett. 114, 251601 (2015), 1503.07114

[12] M. Attems, J. Casalderrey-Solana, D. Mateos, I. Papadimitriou, D. Santos-Oliván, C.F. Sopuerta, M. Triana, M. Zilhão, JHEP 10, 155 (2016), 1603.01254

[13] M. Attems, J. Casalderrey-Solana, D. Mateos, D. Santos-Oliván, C.F. Sopuerta, M. Triana, M. Zilhão, JHEP 01, 026 (2017), 1604 .06439

[14] M. Attems, J. Casalderrey-Solana, D. Mateos, D. Santos-Oliván, C.F. Sopuerta, M. Triana, M. Zilhão, JHEP 06, 154 (2017), 1703.09681

[15] M. Attems, Y. Bea, J. Casalderrey-Solana, D. Mateos, M. Triana, M. Zilhao, JHEP 06, 129 (2017), 1703.02948

[16] R. Critelli, J. Noronha, J. Noronha-Hostler, I. Portillo, C. Ratti, R. Rougemont (2017), 1706.00455

[17] J. Casalderrey-Solana, H. Liu, D. Mateos, K. Rajagopal, U.A. Wiedemann (2011), 1101.0618

[18] M. Bianchi, D.Z. Freedman, K. Skenderis, Nucl. Phys. B631, 159 (2002), hep-th/0112119

[19] P.C. Aichelburg, R.U. Sexl, Gen. Rel. Grav. 2, 303 (1971)

[20] C. Athanasiou, K. Rajagopal, M. Stephanov, Phys. Rev. D82, 074008 (2010), 1006.4636

[21] A. Monnai, S. Mukherjee, Y. Yin, Phys. Rev. C95, 034902 (2017), 1606.00771

[22] P.M. Chesler, M. Lekaveckas, K. Rajagopal, JHEP 10, 013 (2013), 1306.0564

[23] M. Lekaveckas, K. Rajagopal, JHEP 02, 068 (2014), 1311.5577

[24] K. Paech, S. Pratt, Phys. Rev. C74, 014901 (2006), [Erratum: Phys. Rev.C93,no.5,059902(2016)], nucl-th/0604008

[25] P.B. Arnold, C. Dogan, G.D. Moore, Phys. Rev. D74, 085021 (2006), hep-ph/0608012

[26] F. Karsch, D. Kharzeev, K. Tuchin, Phys. Lett. B663, 217 (2008), 0711.0914

[27] S. Ryu, J.F. Paquet, C. Shen, G.S. Denicol, B. Schenke, S. Jeon, C. Gale, Phys. Rev. Lett. 115, 132301 (2015), 1502.01675

[28] J.E. Bernhard, J.S. Moreland, S.A. Bass, J. Liu, U. Heinz, Phys. Rev. C94, 024907 (2016), 1605.03954

[29] M. Alqahtani, M. Nopoush, R. Ryblewski, M. Strickland (2017), 1705.10191

[30] J. Casalderrey-Solana, D. Mateos, W. van der Schee, M. Triana, JHEP 09, 108 (2016), 1607.05273

[31] C. Hoyos, N. Jokela, D. Rodríguez Fernández, A. Vuorinen, Phys. Rev. D94, 106008 (2016), 1609.03480 